\title{
A NON-INVASIVE SINGLE-BUNCH MATCHING AND EMITTANCE MONITOR FOR THE CERN PS BASED ON QUADRUPOLE PICK-UPS
}

\author{
A. Jansson and L. Søby, CERN, Geneva, Switzerland
}

\section{Abstract}

On-line monitoring of beam quality for high brightness beams is only possible using non-invasive instruments. For matching measurements, very few such instruments are available. Therefore, a new type of quadrupole pick-up, sensitive to the beam width, has been developed for the CERN PS. A measurement system consisting of two such pick-ups has been installed in the accelerator. Using the information from these pick-ups, it is possible to determine not only the injection matching in the two planes, but also the emittances. Both measurements use the periodicity of the circular machine to eliminate a number of systematic error sources, while the statistical error is reduced by using data from several turns. The analysis can be done on each bunch separately. This is required because of the PS filling scheme, where each injected bunch comes from a different Booster ring, and therefore can have different properties. After an overview of the measurement system, some of the first measurement results are presented along with a discussion on the measurement error.

\section{INTRODUCTION AND BACKGROUND}

A quadrupole pick-up is a non-invasive device that measures the quadrupole moment

$$
\kappa=\sigma_{x}^{2}-\sigma_{y}^{2}+\bar{x}^{2}-\bar{y}^{2} .
$$

of the transverse beam distribution. Here, $\sigma_{x}$ and $\sigma_{y}$ are the r.m.s. beam dimensions in the $x$ and $y$ directions, while $\bar{x}$ and $\bar{y}$ denote the beam position.

The use of quadrupole pick-ups for emittance measurements was pioneered at SLAC [1], where six pick-ups along the linac were used, and the emittance and Twiss parameters were obtained by solving a matrix equation derived from the known transfer matrices between pick-ups.

In rings, beam size oscillations due to injection mismatch produce sidebands to the revolution frequency harmonics at twice the betatron frequency, $f_{\text {rev }}\left(n \pm 2 q_{h, v}\right)$. The use of quadrupole pick-ups in rings has largely focused on determining the phase and amplitude of these frequency components in the raw pick-up signal[2].

This paper develops the idea behind the SLAC method for use in rings. The quadrupole pick-ups used for this purpose have been specially developed for the PS and optimised to measure the quadrupole moment[3]. Two pickups have recently been installed in consecutive straight sections (numbers 3 and 4 ) of the machine[4]. The optical parameters at the pick-up locations are given in Table 1.

The PS pick-ups measure the evolution of both position and quadrupole moment of each bunch over several hun-
Table 1: Optical parameters at pick-up locations

\begin{tabular}{|c|c|c|c|c|c|}
\hline Name & $\beta_{x}$ & $\beta_{y}$ & $D_{x}$ & $\Delta \mu_{x}$ & $\Delta \mu_{y}$ \\
\hline QPU 03 & $22.0 \mathrm{~m}$ & $12.5 \mathrm{~m}$ & $3.04 \mathrm{~m}$ & \multirow{2}{*}{0.365} & 0.368 \\
QPU 04 & $12.6 \mathrm{~m}$ & $21.9 \mathrm{~m}$ & $2.30 \mathrm{~m}$ & & \\
\hline
\end{tabular}

dred turns. It is therefore possible to subtract the beam position contribution from the measured quadrupole moment, and retain only the beam-size related part. Throughout the rest of this paper, when referring to $\kappa$, it will be assumed that this 'artificial centring' has been performed. Such a corrected measurement is shown in Fig. 1.

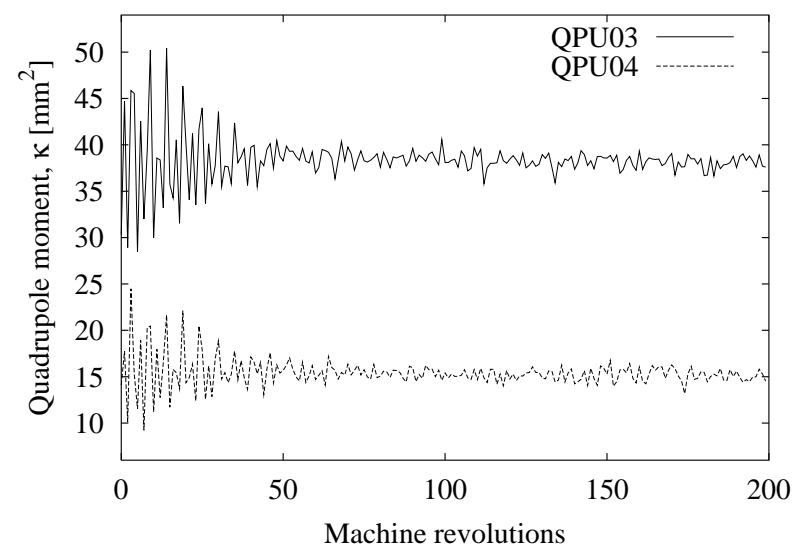

Figure 1: Quadrupole moment $\kappa$ measured with the PS pick-ups immediately after injection. Note the fast filamentation (i.e. damping) of beam size oscillations due to space charge. The constant value after filamentation indicates that the correction for beam position works well.

\section{SIGNAL COMPONENTS}

The quadrupole moment of a beam can be expanded in terms of the optical parameters as

$$
\kappa=\sigma_{x}^{2}-\sigma_{y}^{2}=\varepsilon_{x} \beta_{x}-\varepsilon_{y} \beta_{y}+\sigma_{p}^{2} D_{x}^{2}-\sigma_{p}^{2} D_{y}^{2}
$$

assuming linear optics with no coupling between planes. If the beam is initially mismatched in terms of Twiss functions or dispersion, the value of $\kappa$ will vary with the number of machine revolutions $n$ performed as[5]

$$
\begin{gathered}
\kappa(n)=\bar{\beta}_{x}\left(\varepsilon_{x}+\Delta \varepsilon_{x}\right)-\bar{\beta}_{y}\left(\varepsilon_{y}+\Delta \varepsilon_{y}\right)+\bar{D}_{x}^{2} \sigma_{\mathrm{p}}^{2} \\
+\bar{\beta}_{x} \varepsilon_{x} \delta_{\beta_{x}} \cos \left(2 \nu_{x} n-\phi_{\beta_{x}}\right)+\bar{\beta}_{x} \sigma_{\mathrm{p}}^{2} \delta_{D_{x}}^{2} \cos \left(2 \nu_{x} n-2 \phi_{D_{x}}\right) \\
-\bar{\beta}_{y} \varepsilon_{y} \delta_{\beta_{y}} \cos \left(2 \nu_{y} n-\phi_{\beta_{y}}\right)-\bar{\beta}_{y} \sigma_{\mathrm{p}}^{2} \delta_{D_{y}}^{2} \cos \left(2 \nu_{y} n-2 \phi_{D_{y}}\right) \\
+\sqrt{\bar{\beta}_{x}} \sigma_{\mathrm{p}}^{2} \bar{D}_{x} \delta_{D_{x}} \cos \left(\nu_{x} n-\phi_{D_{x}}\right)
\end{gathered}
$$


Here, barred parameters refer to properties of the lattice, and $\nu_{x, y}=2 \pi q_{x, y}$. The terms have been ordered so that each line corresponds to one oscillation frequency. Starting from the bottom, the fourth line is a signal at the horizontal betatron frequency, which is due to dispersion matching. This mismatch is parametrised by the vector

$$
\vec{\delta}_{D_{x}}=\left(\begin{array}{c}
\frac{\Delta D_{x}}{\sqrt{\bar{\beta}_{x}}} \\
\sqrt{\bar{\beta}_{x}} \Delta D_{x}^{\prime}+\bar{\alpha}_{x} \frac{\Delta D_{x}}{\sqrt{\bar{\beta}_{x}}}
\end{array}\right)
$$

There is no corresponding signal at the vertical betatron frequency due to the absence of vertical lattice dispersion.

The middle lines of Eq. (3) are signal components at twice the horizontal and vertical betatron frequencies. They arise from both dispersion and betatron mismatch. The betatron mismatch is parametrised by

$$
\vec{\delta}_{\beta_{x}}=\left(\begin{array}{c}
\frac{\beta_{x}}{\bar{\beta}_{x}}-\frac{\bar{\beta}_{x} \gamma_{x}+\bar{\gamma}_{x} \beta_{x}-2 \bar{\alpha}_{x} \alpha_{x}}{2} \\
\frac{\bar{\alpha}_{x} \beta_{x}-\bar{\alpha}_{x} \bar{\beta}_{x}}{\bar{\beta}_{x}}
\end{array}\right) \approx\left(\begin{array}{c}
\frac{\Delta \beta_{x}}{\bar{\beta}_{x}} \\
\bar{\alpha}_{x} \frac{\Delta \beta_{x}}{\beta_{x}}-\Delta \alpha_{x}
\end{array}\right)
$$

where the last approximation is valid for small mismatch.

The first line of Eq. (3) contains the constant terms, which correspond to the steady state that is reached when the oscillating components have damped away. The steady state (filamented) emittance is given by

$$
\begin{gathered}
\varepsilon_{x}+\Delta \varepsilon_{x}=\varepsilon_{x} \frac{1}{2}\left(\bar{\beta}_{x} \gamma_{x}+\bar{\gamma}_{x} \beta_{x}-2 \bar{\alpha}_{x} \alpha_{x}\right)+ \\
+\sigma_{\mathrm{p}}^{2} \frac{\left(\Delta D_{x}\right)^{2}+\left(\bar{\beta}_{x} \Delta D_{x}^{\prime}+\bar{\alpha}_{x} \Delta D_{x}\right)^{2}}{\bar{\beta}_{x}} \approx \\
\approx \varepsilon_{x}+\varepsilon_{x} \frac{\delta_{\beta_{x}}^{2}}{2}+\sigma_{\mathrm{p}}^{2} \frac{\delta_{D_{x}}^{2}}{2}
\end{gathered}
$$

where, again, the last approximation is valid for small betatron mismatch.

There is also an emittance increase due to mis-steering at injection that is not included here, since normally beam size oscillations filament much faster than beam position oscillations due to the incoherent space charge tune shift.

\section{EMITTANCE MEASUREMENT}

If the circulating beam is stable, the two pick-up signals are the same each turn and given by

$$
\begin{aligned}
& \kappa_{1}=\epsilon_{x} \bar{\beta}_{x 1}-\epsilon_{y} \bar{\beta}_{y 1}+\bar{D}_{x 1}^{2} \sigma_{\mathrm{p}}^{2} \\
& \kappa_{2}=\epsilon_{x} \bar{\beta}_{x 2}-\epsilon_{y} \bar{\beta}_{y 2}+\bar{D}_{x 2}^{2} \sigma_{\mathrm{p}}^{2}
\end{aligned}
$$

If the momentum spread is known, the system of equations can be solved for the emittances, since

$$
\frac{\bar{\beta}_{x 1}}{\bar{\beta}_{y 1}} \neq \frac{\bar{\beta}_{x 2}}{\bar{\beta}_{y 2}}
$$

Thus, measuring the emittance of a stable circulating beam with quadrupole pick-ups is rather straightforward.
The statistical error due to random fluctuations in the measurement of $\kappa$ are small (less than $0.5 \mathrm{~mm}^{2}$ r.m.s.), and can be further reduced by averaging over many consecutive beam passages. The dominant errors will therefore be systematic, coming from offsets in the pick-ups and errors in the beta functions, lattice dispersion and momentum spread. The pick-up offsets are known from measurements in the laboratory. Furthermore, by comparing the amplitude of position oscillations as measured by the two pickups the ratios $\beta_{x_{1}} / \beta_{x_{2}}$ and $\beta_{y_{1}} / \beta_{y_{2}}$ can be determined.

The main uncertainty is thus the absolute value of the beta function, as for any other measurement of stable-beam emittance (e.g. with a wire-scanner). The accuracy can therefore be expected to be comparable to these methods. An emittance measurement using the pick-up system is shown in Fig. 2, and compares well with wire-scanner results.
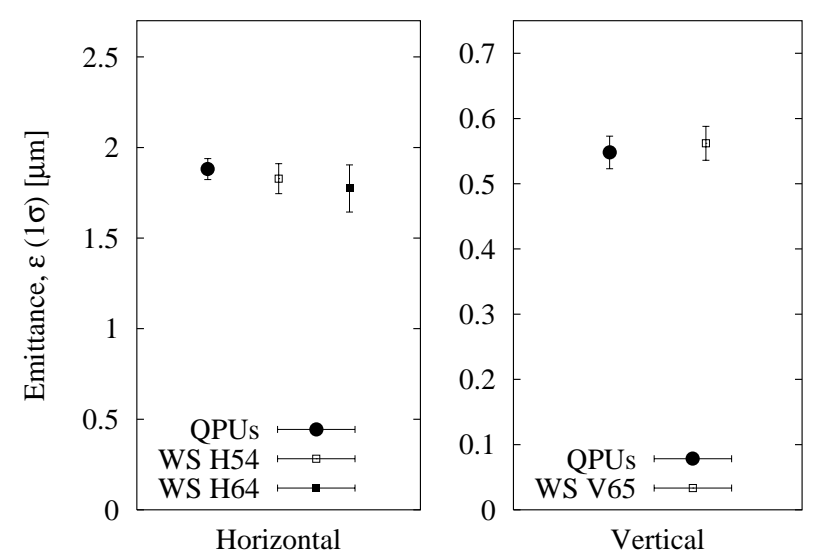

Figure 2: Filamented emittance of a proton beam measured with quadrupole pick-ups (QPU) and wire-scanner (WS). There is a good agreement. The error bar is the standard deviation for 10 measurements.

\section{MATCHING MEASUREMENT}

At injection, the quadrupole moment of the beam is not constant if the beam is mismatched. To determine the parameters of the injected beam, the SLAC method can be directly applied if the quadrupole moment is measured on a single-pass basis.

An advantage in a ring, with respect to a linac, is that each pick-up can be used several times on the same bunch, so that six separate pick-ups are not necessary (at least two pick-ups are however needed to disentangle the horizontal and vertical contributions). This reduces both the cost and the systematic error sources. In a ring, the matrix equation to be solved is a function of only a few parameters, namely the Twiss parameters at the pick-ups, the machine tunes, and the pick-up phase separations. The tunes and the pickup phase separations can be determined experimentally by measuring phase differences and frequency of beam position oscillations in the two planes. Similarly, as pointed out previously, in each plane the ratio of beta functions at the 
two pick-ups can be determined.

However, space charge effects detune the beam width oscillation frequencies. The tunes, as measured from position oscillations, therefore only approximatively describe beam width oscillations. To account for this effect, instead of solving a system of equations derived for a fixed tune, the expression in Eq. (3) is fitted to the measured data, with a variable tune (the phase advances between pick-ups are insensitive to space charge effects since they are so close).

In such a fit, it is also straightforward to include the effect of dispersion mismatch, something that was not treated in the original SLAC method.

The horizontal dispersion mismatch is determined from the oscillation it induces at the betatron frequency. Due to the absence of vertical lattice dispersion, it is not possible to separate betatron and dispersion mismatch in the vertical plane, but a large vertical dispersion mismatch is not expected. Note that the beam size oscillations due to dispersion mismatch are also detuned by space charge, and therefore the effect of dispersion can not, in general, be measured separately (by changing the energy of the beam and measuring the coherent response) and subtracted from the beam size.

The new free parameters (tunes and dispersion) require more data points, bringing the total necessary to ten (five machine turns with two pick-ups). Some more points should be used in the fit to control and quantify the error, but using too many machine turns would deteriorate the result, since the signal is damped. Fortunately, due to the physics of the filamentation process, the decay is not exponential, but rather follows a Gaussian-like curve. The oscillation amplitude is therefore almost unchanged by the filamentation process over the first couple of turns.

As in the previous case, random errors are suppressed by using data from several turns, so systematic errors arising from erroneous input parameters are the main issue for the accuracy of the measurement. The fit result can be unstable

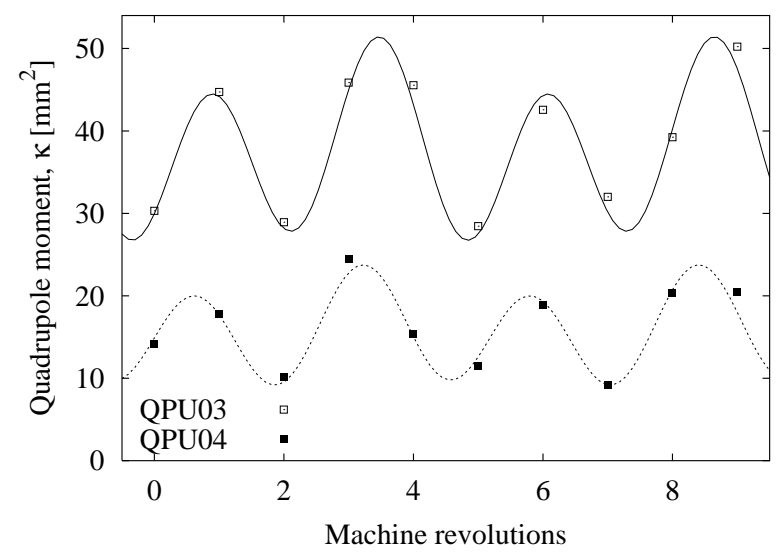

Figure 3: Theoretical expression for the quadrupole moment fitted to measured data. Ten turns (20 data points) were used to determine the ten free parameters (emittances, betatron and dispersion mismatches, and the tunes). for tunes close to 0.25 , and for $q_{x}=q_{y}=0.25$ the method does not work at all (which is not a great problem since there are beam dynamics reasons for avoiding these tunes).

An example is shown in Fig. 3. Comparative measurements have been done using a turn-by-turn SEM grid[6], which is destructive and can only be used in rare dedicated machine development sessions. The results so far have been encouraging, but is has not yet been possible to make a quantitative comparison.

\section{POSSIBLE EXTENSIONS}

In the PS, there are only two sets of locations (even and odd straight section) with significantly different optical parameters. This is why only two pick-ups were installed. In another machine a third pick-up, suitably located, would enable independent measurement of the momentum spread, by adding a third line to Eq. (7).

This would be possible for example in the SPS, by installing two pick-ups in a dispersion-free straight section (one at large horizontal beta, and one at large vertical beta) and one pick-up in an arc, where the dispersion is large.

\section{CONCLUSIONS}

A system for non-invasive measurement of emittance and matching has been installed in the PS. The initial experience with this system is good, and comparison with other instruments has given encouraging results. Further tests on beams with varying characteristics will be performed. The next step is to attempt to correct the measured mismatches, with the ultimate aim of making the system an operational tool, linked to the existing automatic beam correction system for betatron and dispersion matching[7].

\section{REFERENCES}

[1] R.H. Miller et al, "Non-Invasive Emittance Monitor", Proc. 12th Int. Conf. on High Energy Accel., Batavia, IL, 1983.

[2] V. Chohan et al, "Measurement of Coherent Quadrupole Oscillations at Injection into the Antiproton Accumulator", Proc. European Part. Acc. Conf., Nice, France, 1990.

[3] A. Jansson, D.J. Williams, "A New Optimised Quadrupole Design using Magnetic Coupling”, to be published in Nucl. Instr. Meth. in Phys. Res. A

[4] A. Jansson, L. Søby, D.J. Williams, "Design of a Magnetic Quadrupole Pick-up for the CERN PS', DIPAC'01, Grenoble, France, 2001

[5] A. Jansson, "Non-Invasive Measurement of Emittance and Optical Parameters for High-Brightness Hadron Beams in a Synchrotron", Dissertation, Stockholm University, Stockholm, Sweden, 2001

[6] M. Benedikt et al, "Injection Matching Studies using Turn by Turn Beam Profile Measurement in the CERN PS", DIPAC'01, Grenoble, France, 2001

[7] M. Giovannozzi, A. Jansson, M. Martini, "Simultaneous Matching of Dispersion and Twiss Parameters in a Transfer Line”, ABS'98, Geneva, Switzerland, 2001 